\title{
Prognostic factor for the response to intravitreal bevacizumab treatment in central serous chorioretinopathy patients.
}

Chunghyun Lee

Gangneung Asan Hospital

Yu Jeong Kim

Gangneung Asan Hospital

Soo Geun Joe

$J$ 's retina clinic

Sungjae Yang ( $\nabla$ sungjaeyang@gmail.com )

$J$ 's retina clinic

Research article

Keywords: Central serous chorioretinopathy, Bevacizumab, Subfovealchoroidal thickness

Posted Date: October 1st, 2019

DOI: https://doi.org/10.21203/rs.2.15383/v1

License: (c) (i) This work is licensed under a Creative Commons Attribution 4.0 International License.

Read Full License 


\section{Abstract}

Objective To evaluate prognostic factors associated with good response to intravitreal bevacizumab (IVB) treatment in central serous chorioretinopathy (CSC) patients.

Methods We retrospectively reviewed ninety-four eyes of 94 CSC patients who received intravitreal bevacizumab injection(IVB) $(0.05 \mathrm{ml}, 1.25 \mathrm{mg})$ as a first line treatment. Patients were divided into two groups as good responders and poor responders based on optical coherence tomography (OCT) findings 1 month after the first injection of intravitreal bevacizumab. Good responders were defined as complete resolution of subretinal fluid (SRF) on OCT 1 month after IVB. We compared baseline visual acuity, duration of symptoms, angiographic findings on fluorescein angiography, central retinal thickness (CRT), macular volume (MV),subfoveal choroidal thickness (SFCT) between two groups.

Results 42 eyes were included as good responder and 52 eyes as poor responders. Mean age was 47.8 \pm 7.4 year old ingood responders and $50.6 \pm 7.8$ year old in poor responders. Baseline visual acuity ( $\log M A R)$ was better in good responders than poor responders, $0.19 \pm 0.19$ and $0.29 \pm 0.19$ respectively $(p=0.015)$. SFCT was significantly thicker in good responders than poor responders $419.7 \pm 103.2 \nabla$ and $376.0 \pm 99.0 \otimes$ respectively $(p=0.040)$. Prevalence of chronic cases was higher in poor responders $(33 / 52)$ than good responders (15/42), ( $p=0.012)$. There was no statistical significance in age, sex, CRT, MVbetween two groups. Conclusion Intravitreal injection of bevacizumab can be potential option for the treatment of CSC patients, especially in case of recent onset, better baseline visual acuity with thicker choroid on OCT.

\section{Background}

Central serous chorioretinopathy (CSC) is characterized by localized serous sensory detachment of neurosensory retina. ${ }^{1}$ In the majority of patients, serous retinal detachment resolves spontaneously; however, some patients with CSC develop progressive visual loss from persistent serous retinal detachment, cystoid macular degeneration or retinal pigment epithelium(RPE)decompensation. ${ }^{2}$ The pathogenesis remains unclear, but is associated with hyperpermeability of choroid and retinal pigment epithelial dysfunction. ${ }^{3,4}$ There are no gold standard treatment for CSC, focal laser photocoagulation, photodynamic therapy (PDT), intravitreal injections of anti-vascular endothelial growth factor (VEGF), corticosteroid antagonists, and acetazolamide have been used to promote the resorption of subretinal fluid in CCS patients. ${ }^{5-9}$ Intravitreal bevacizumab (IVB), a recombinant humanized monoclonal anti-VEGF antibody, has been reported to be effective and safe for the treatment of CSC patients. ${ }^{10,11}$ In previous study, Jung et al. suggest that IP-10 and VEGF may contribute to the pathogenesis of CSC. And intravitrealbevacizumab was helpful in some of the patients with CSC, who showed higher levels of VEGF in the aqueous humor. ${ }^{12}$ There were recent studies regarding clinical characteristics of CSC patients who had better response to intravitreal injection of bevacizumab. ${ }^{13},{ }^{14}$ Butit is not clearly known which factors would predict favorable or unfavorable response to intravitreal bevacizumab treatment in CSC patients. 
Herein, we investigated the prognostic clinical factors associated with initial response to intravitreal injection of bevacizumab in treatment naïve CSC patients.

\section{Methods}

Study design and participants

We retrospectively reviewed the medical record was of CSC patients who were treated with intravitreal injection of bevacizumabunder diagnosis of CSC at GangneungAsan Hospital, Gangneung. Korea, from July, 2013 to April 2018.

The study was approved by the Institutional Review Board of GangneungAsan Hospital and this study followed the tenets of the Declaration of Helsinki.

We included treatment naïve CSC patients who showed leakage on fluorescein angiography (FA) and serous sensory retinal detachment on OCT. Exclusion criteria were as follows: (1)patients who received any previous treatment for CSC such as focal laser photocoagulation, PDT, systemic carbonic anhydrase inhibitors, (2) steroid induced CSC patients, (3) patients who had co-existing macular pathologies such as diabetic retinopathy, severe epiretinal membrane or age related macular degeneration, (4) patients with spherical equivalence $>+3.00$ or $<-6.00$ Diopter.

Ophthalmic examinations

The patients underwent comprehensive ophthalmic examinations, including best corrected visual acuity (BCVA), dilated fundus examination, FA and spectral domain optical coherence tomography (SD-OCT) with enhanced depth imaging technique (EDI-OCT).

BCVA results were converted to the logarithm of the minimum angle of resolution (logMAR) scale for the comparison.FA (Topcon TRC-50IX,Tokyo, Japan) was performed, we categorized the patients into focal leakage type and diffuse leakage type based on FA findings(Figure 1). Heidelberg Spectralis (Heidelberg Engineering) was used to obtain SD-OCT images of the macula using a custom $25^{\circ} \times 25^{\circ}$ volume acquisition protocol to obtain a set of high-speed scans from each eye. Using the protocol, 25 crosssectional B-scan images were obtained, each of which was composed of 512 A-scans. Choroidal images were obtained using the EDI technique. Subfovealchoroidal thickness (SFCT) was vertically measured from the outer border of the RPE to the inner border of the sclera by two retina specialists (Joe and Yang) using calipers and Heidelberg Eye Explorer software (Figure2). The mean data of two different measurements were used for comparison in this study. Central retinal thickness (CRT)and macular volume (MV)was obtained automatically and manually adjusted when the reference line was deviated.

Six weeks after intravitreal injection of bevacizumab, we performed BCVA, fundus examination and OCT. We compared ophthalmic findings and variables before IVB and 1 month after IVB to investigate the parameters which were associated with initial response. We divided the patients into two groups based on the status of subretinal fluid on OCT 1 month after IVB. Good responders were defined who showed 
complete resorption of subretinal fluid on OCT, whereas poor responders showed incomplete resorption of SRF on OCT 1 month after initial IVB(Figure 3).

We compared BCVA, duration of symptoms, SFCT, CRT, MV and fluorescein leakage type on FA.

\section{Statistics}

The independent t-test was used to compare BCVA, SFCT, CRT and MV between bevacizumab good responders and poor responders. The Fisher's exact test was used to analyze the relationship between each group (responsive or nonresponsive) and sex, acute or chronic status, FA findings (focal leakage or diffuse leakage). Clinical characteristics were compared between the bevacizumab good responders and poor responders with independent t-test IBM SPSS ver. 21.0 (IBM Corp., Armonk, NY, USA) was used to perform all analyses, and $p<0.05$ was considered statistically significant.

\section{Results}

Ninety four eyes of 94 patients were included in this study. Forty two patients were included in good responders and 52 were in poor responders. Table 1 shows demographic characteristics and ophthalmic variables of the population in this study. Mean age of good responders was $47.8 \pm 7.4$ year-old and that of poor responders was $50.6 \pm 7.8$, good responders were younger but there was no statistical significance. Baseline BCVA was significantly better in good responders than poor responders, $0.20 \pm 0.20$ (logMAR)and $0.30 \pm 0.20$ (logMAR). Good responders showed thicker SFCT $(419.7 \pm 103.2 \varnothing)$ than poor responders

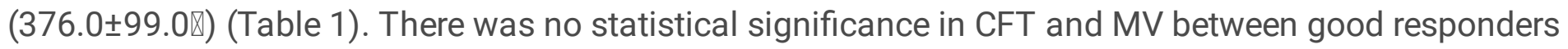
and poor responders $(p=0.998$ and $p=0.498$, respectively) at initial presentation. We defined acute CSC when the symptom duration was less than three months and chronic when the symptom duration was longer than 3 months. We investigated the prevalence of acute and chronic cases in both groups and compared. Prevalence of acute cases were more in good responders thanpoor responders (24 out of 42 and 19 out of $52, p=0.029$ ). There were no significant differences in FA leakage type and sex ratio between both groups $(p=0.198$ and $p=0.776$, respectively, Table 1$)$.

1 month after IVB, CRT was thinner and MV was less in good responders than poor responders, those results were statistically significant ( $p=0.000$ vs $p=0.001$ respectively). Post-IVB BCVA was significantly better in good responders, also $(p=0.000)$.

We investigated the correlation between BCVA and CRT, or MV. There was no significant correlation between BCVA and CRT, or MV ( $p=0.918$ and 0.490 respectively, Table 1$)$ at initial presentation. Two studies commonly showed thicker choroid responded better to IVB in CSC patients.

\section{Discussion}

Anti-VEGF intravitreal injection is one of major option for the treatment of CSC, and previously published articles were focused on chronic and recurrent cases. Most studies showed favorable outcomes, but not 
all cases of chronic and recurrent CSC patients respond to IVB well. ${ }^{10,11,15}$

The aim of this study was to investigate the prognostic factor for IVB in treatmentnaive CSC patients and there were few studies regarding this topic. Kim et al. published bevacizumab responders showed significantly thicker SFCH at baseline and great reduction in SFCT after intravitreal injection of bevacizumab. ${ }^{14}$ In this study, authors compared only choroid thickness variation between bevacizumab responders and poor responders with thirty eyes of 30 patients and the relationship between late indocyanine green angiography (ICGA) findings and the responsiveness to IVB. The other study showed CSC patients with thicker choroid and better initial visual acuity was correlated with better response to IVBtreatment in 42 chronic recurrent CSC patients. ${ }^{13}$ The result of this study was similar with our result, but only included chronic CSC cases. Traditionally, abnormality of barrier function in RPE was considered as a primary cause in CSC patients. With advancement in imaging modalities such as ICGA, abnormal choroidal vasculature was considered as a main cause. ${ }^{16,17}$ Jirarattanasopa et al. showed the association between choroidal thickness and choriovascular permeability on ICGA. ${ }^{18}$ This indirectly means that the role of anti-VEGF treatment in CSC patients would be better in thicker choroid which was more vascular permeable. Also those results strengthened the rationale for anti-VEGF treatment in CSC patients.

Our study has some limitation of retrospective design. Furthermore, we did not include an untreated control group of CSC eyes. It is possible that some good responder patients experienced spontaneous improvement or resolution. However, we minimized this possibility by excluding patients with symptom duration that was $<3$ months. Further studies should be prospectively designed and include an untreated control group.

The sample size was relatively larger than previous studies.We treated IVB injection inacute CSC patients who needed rapid visual recovery when they could not have focal laser treatment. This was the main difference from previous studies, which have been used IVB mostly in chronic or recurrent CSC cases.

\section{Conclusions}

CSC patients with relatively acute onset, thicker subfovealchoroidand better initial VA had shown better response to IVB in our study. Based on our results, IVB can be a primary treatment for CSC eyes with thicker subfoveal choroidand good VA even in acute phase.

\section{List Of Abbreviations}

IVB: Intravitreal bevacizumab

CSC: Central serous chorioretinopathy

PDT: Photodynamic therapy 
logMAR: Logarithm of minimum angle of resolution

VEGF: Vascular endothelial growth factor

FA: Fluorescein angiography

SFCT: Subfoveal choroidal thickness

CRT: Central retinal thickness

MV: Macular volume

\section{Declarations}

-Ethics approval and consent to participate

The study was approved by the Institutional Review Board of GangneungAsan Hospital and this study followed the tenets of the Declaration of Helsinki.

-Availability of data and material

All the data supporting the conclusions of this article are included within the article and its figures.

- Consent for publication

Written informed consent was obtained from the patient for publication of this case report and any accompanying images. A copy of the written consent is available for review by the Editor of this journal.

- Competing interests

The author has no financial or conflicting interest.

- Funding

No funding was obtained for this study.

- Authors' contributions

Dr. Lee is the first author who wrote this article and 2 nd and 3rh authors contributed to data anlysis.

Corresponding author conducted to design this study.

- Acknowledgements

none 


\section{References}

1.Daruich A, Matet A, Dirani A, Bousquet E, Zhao M, Farman N, Jaisser F and Behar-Cohen F. Central serous chorioretinopathy: Recent findings and new physiopathology hypothesis. Progress in retinal and eye research. 2015;48:82-118.

2.Loo RH, Scott IU, Flynn HW, Jr., Gass JD, Murray TG, Lewis ML, Rosenfeld PJ and Smiddy WE. Factors associated with reduced visual acuity during long-term follow-up of patients with idiopathic central serous chorioretinopathy. Retina (Philadelphia, Pa). 2002;22:19-24.

3.Marmor MF. New hypotheses on the pathogenesis and treatment of serous retinal detachment. Graefe's archive for clinical and experimental ophthalmology = Albrecht von Graefes Archiv fur klinische und experimentelle Ophthalmologie. 1988;226:548-52.

\section{Prunte $\mathrm{C}$ and Flammer $\mathrm{J}$. [Circulatory disorders of the choroid in patients with central serious chorioretinopathy]. Klinische Monatsblatter fur Augenheilkunde. 1996;208:337-9.}

\section{Burumcek E, Mudun A, Karacorlu S and Arslan MO. Laser photocoagulation for persistent central serous retinopathy: results of long-term follow-up. Ophthalmology. 1997;104:616-22.}

6.Nielsen JS and Jampol LM. Oral mifepristone for chronic central serous chorioretinopathy. Retina (Philadelphia, Pa). 2011;31:1928-36.

7.Pikkel J, Beiran I, Ophir A and Miller B. Acetazolamide for central serous retinopathy. Ophthalmology. 2002;109:1723-5. 
8.Ruiz-Moreno JM, Lugo FL, Armada F, Silva R, Montero JA, Arevalo JF, Arias L and Gomez-Ulla F. Photodynamic therapy for chronic central serous chorioretinopathy. Acta ophthalmologica. 2010;88:371-6.

9.Torres-Soriano ME, García-Aguirre G, Kon-Jara V, Ustariz-Gonzáles O, Abraham-Marín M, Ober MD and Quiroz-Mercado H. A pilot study of intravitreal bevacizumab for the treatment of central serous chorioretinopathy (case reports). Graefe's archive for clinical and experimental ophthalmology = Albrecht von Graefes Archiv fur klinische und experimentelle Ophthalmologie. 2008;246:1235-1239.

10.Artunay O, Yuzbasioglu E, Rasier R, Sengul A and Bahcecioglu H. Intravitreal bevacizumab in treatment of idiopathic persistent central serous chorioretinopathy: a prospective, controlled clinical study. Current eye research. 2010;35:91-8.

\section{Huang WC, Chen WL, Tsai YY, Chiang CC and Lin JM. Intravitreal bevacizumab for treatment of chronic central serous chorioretinopathy. Eye (London, England). 2009;23:488-9.}

\section{Jung SH, Kim KA, Sohn SW and Yang SJ. Cytokine levels of the aqueous humour in central serous chorioretinopathy. Clinical \& experimental optometry. 2014;97:264-9.}

13.Kim GA, Rim TH, Lee SC, Byeon SH, Koh HJ, Kim SS and Lee CS. Clinical characteristics of responders to intravitreal bevacizumab in central serous chorioretinopathy patients. Eye (London, England). 2015;29:732-40; quiz 741.

14.Kim DY, Joe SG, Yang SJ, Lee JY, Kim JG and Yoon YH. The association between choroidal thickness variations and response to intravitreal bevacizumab in central serous chorioretinopathy. Korean journal of ophthalmology: KJO. 2015;29:160-7. 
15.Shin KH, Kim JH, Cho SW, Lee TG, Kim CG and Kim JW. Efficacy of Intravitreal Bevacizumab for Recurrent Central Serous Chorioretinopathy in Patients Who Had Previously Responded Well to the Same Therapy. Journal of ocular pharmacology and therapeutics: the official journal of the Association for Ocular Pharmacology and Therapeutics. 2016;32:425-30.

16.Dohrmann J, Lommatzsch A, Spital G and Pauleikhoff D. [Pathogenesis of central serous chorioretinopathy: angiographic and electrophysiological studies]. Der Ophthalmologe: Zeitschrift der Deutschen Ophthalmologischen Gesellschaft. 2001;98:1069-73.

17.Yannuzzi LA, Slakter JS, Gross NE, Spaide RF, Costa D, Huang SJ, Klancnik JM, Jr. and Aizman A. Indocyanine green angiography-guided photodynamic therapy for treatment of chronic central serous chorioretinopathy: a pilot study. Retina (Philadelphia, Pa). 2003;23:288-98.

18.Jirarattanasopa P, Ooto S, Tsujikawa A, Yamashiro K, Hangai M, Hirata M, Matsumoto A and Yoshimura N. Assessment of macular choroidal thickness by optical coherence tomography and angiographic changes in central serous chorioretinopathy. Ophthalmology. 2012;119:1666-78.

\section{Tables}

Table 1 Comparision of characteristics between good and poor responders. 


\begin{tabular}{|c|c|c|c|}
\hline & Good responders $(n=42)$ & Poor responders $(n=52)$ & $P$ value \\
\hline Age (year) & $47.8 \pm 7.4$ & $50.6 \pm 7.8$ & $0.080 *$ \\
\hline Male : Female & $36: 6$ & $39: 13$ & $0.302^{\dagger}$ \\
\hline Duration & $24: 18$ & $18: 34$ & $0.029^{\dagger}$ \\
\hline \multicolumn{4}{|l|}{ Acute : Chronic } \\
\hline FA leakage & $19: 23$ & $22: 30$ & $0.776^{\dagger}$ \\
\hline \multicolumn{4}{|l|}{ Focal :Diffuse } \\
\hline \multicolumn{4}{|l|}{ Baseline } \\
\hline BCVA (logMAR) & $0.20 \pm 0.20$ & $0.30 \pm 0.20$ & $0.015^{*}$ \\
\hline SFCT ( () & $419.7 \pm 103.2$ & $376.0 \pm 99.0$ & $0.040 *$ \\
\hline CRT ( () & $431.6 \pm 92.0$ & $431.9 \pm 102.8$ & $0.988^{*}$ \\
\hline $\mathrm{MV}\left(\mathrm{mm}^{3}\right)$ & $9.7 \pm 1.3$ & $9.9 \pm 1.1$ & $0.478^{*}$ \\
\hline \multicolumn{4}{|l|}{ Post IVB } \\
\hline BCVA (logMAR) & $0.11 \pm 0.14$ & $0.24 \pm 0.20$ & $0.000^{*}$ \\
\hline CRT ( () & $255.2 \pm 26.9$ & $346.9 \pm 82.4$ & $0.000^{*}$ \\
\hline $\mathrm{MV}\left(\mathrm{mm}^{3}\right)$ & $8.9 \pm 0.7$ & $9.4 \pm 0.9$ & $0.001 *$ \\
\hline
\end{tabular}

BCVA=Best corrected visual acuity, SFCT=Subfovealchoroidal thickness

$\mathrm{CRT}=$ Central retinal thickness, $\mathrm{MV}=$ Macular volume, $\mathrm{IVB}=$ Intravitrealbevacizumab

*Student $\mathrm{T}$ test, ${ }^{\dagger}$ Chi-square test

\section{Figures}




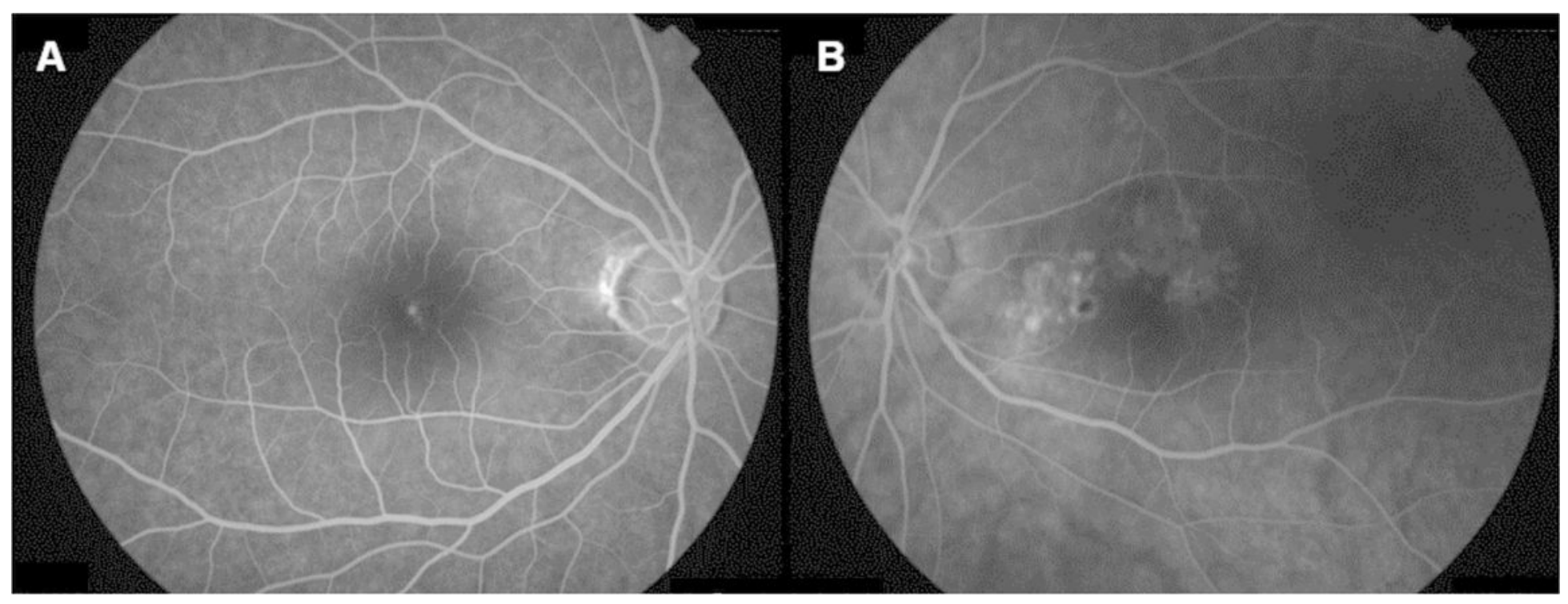

\section{Figure 1}

Fluorescein angiography findings in central serous chorioretinopathy. (A) Focal leakage type was defined as the presence of well-defined homogeneous spots. (B) Diffuse leakage type was defined as the presence of an area with hyperfluorescence without focal well-definded spots

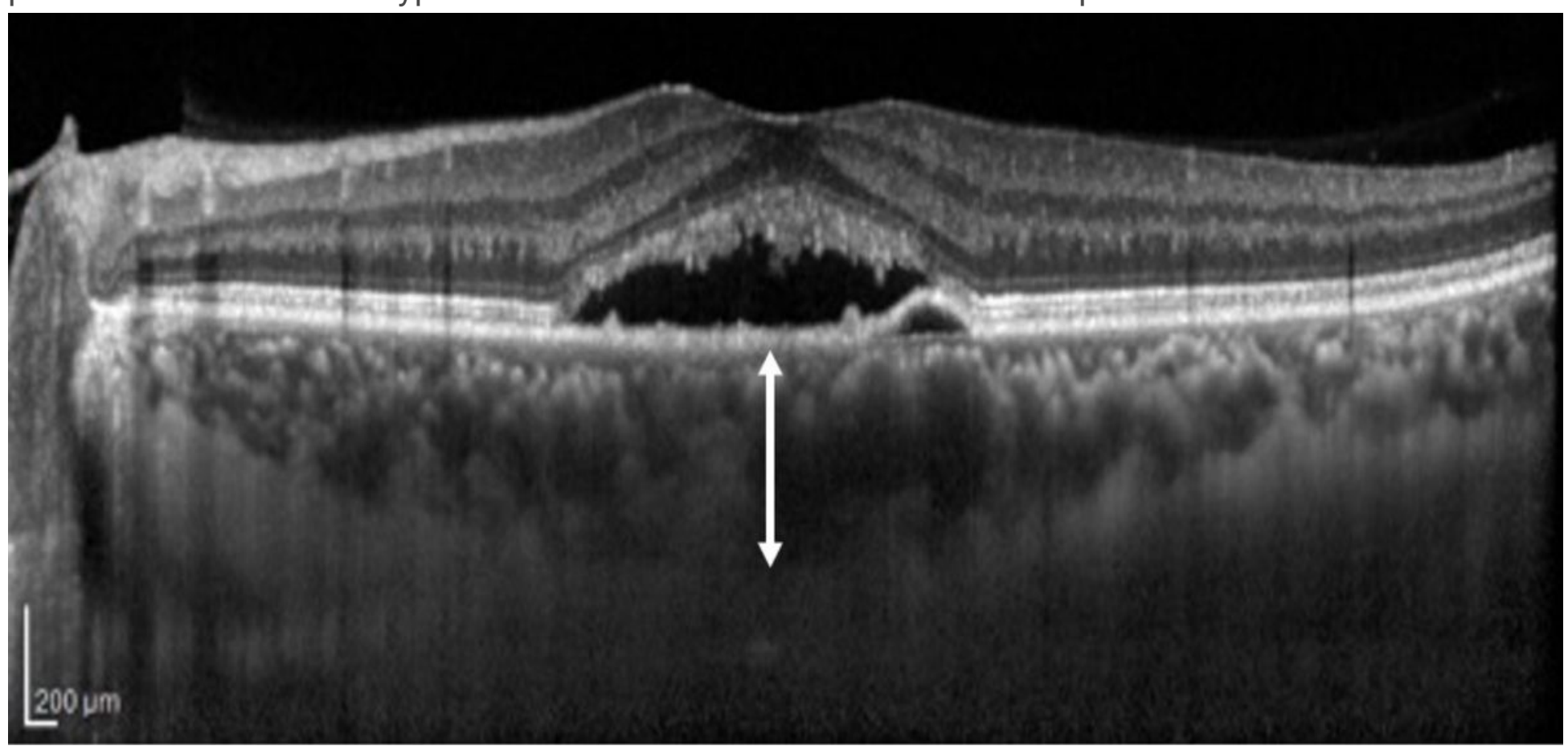

Figure 2

Measurement of SFCT on EDI-OCT image. SFCT was vertically measured from the outer border of the retinal pigment epithelium to the inner border of the sclera. 


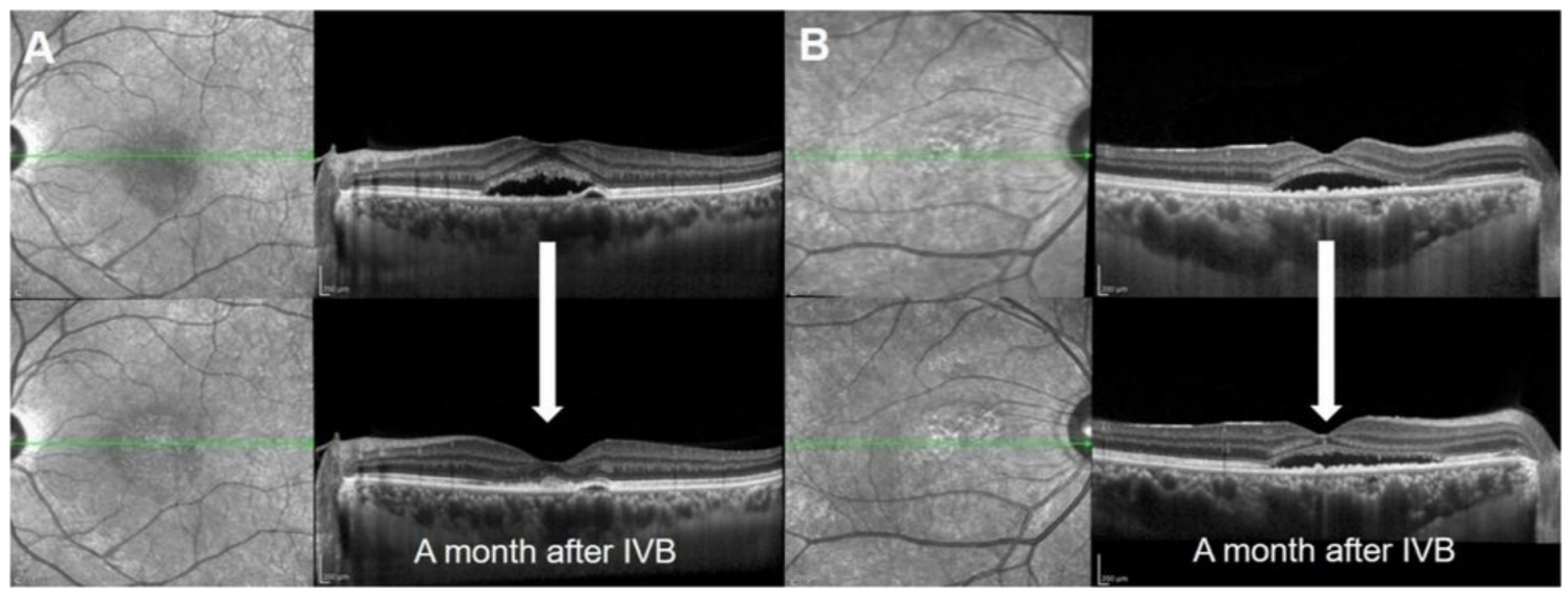

\section{Figure 3}

Response to initialIVBin central serous chorioretinopathy. (A) Good responder was defined as the complete resorption of SRF on OCT after 1 month IVB (B) Poor response was defined as the incomplete resorption of SRF on OCT after 1 month IVB 